\title{
Hydroxymethylation changes during early embryonic development in zebrafish
}

Artem Nedoluzhko

Faculty of Biosciences and Aquaculture

Nord University

Bodø, Norway

artem.nedoluzhko@nord.no

Ioannis Konstantinidis

Faculty of Biosciences and Aquaculture

Nord University

Bodø, Norway

ioannis.konstantinidis@nord.no

\author{
Paula Berrutti \\ Faculty of Biosciences and Aquaculture \\ Nord University \\ Bodø, Norway \\ paula.d.berrutti@nord.no
}

Igor Babiak

Faculty of Biosciences and Aquaculture Nord University

Bodø, Norway

igor.s.babiak@nord.no
Igo Guimarães

Departamento de Produção Animal

Universidade Federal de Goiás

Goias, Brazil

igoguimaraes@ufg.br

Jorge M.O. Fernandes

Faculty of Biosciences and Aquaculture

Nord University

Bodø, Norway

jorge.m.fernandes@nord.no
Abstract - A large number of studies in organisms across the eukaryotic phyla have shown that DNA 5-methylcytosine $(5 \mathrm{mC})$ modification is one of many mechanisms that suppress gene expression.

Keywords - zebrafish, Danio rerio, embryo, development, hydroxymethylation

Cytosine hydroxymethylation has been described during the past years; this DNA modification is increasingly recognized as an important component of epigenetic regulation in eukaryotes.

In the present study, we investigated if hydroxymethylation may be involved in fish embryonic development and demonstrated for the first time at a genomewide level and single nucleotide resolution the hydroxymethylome changes during zebrafish (Danio rerio) embryogenesis from one-cell stage to hatching. DNA hydroxymethylation was profiled by reduced representation hydroxymethylation profiling (RRHP), as shortly described in Fig. 1.

Taken together with recently published data on 5methylcytosine $(5 \mathrm{mC})$ modification events in $D$. rerio, our data unveil a new role for DNA hydroxymethylation in epigenetic regulation of fish embryonic development.

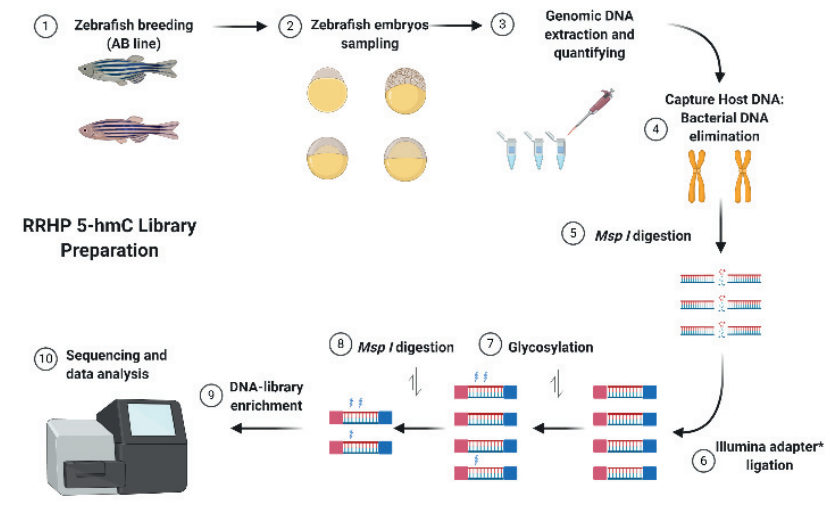

Fig. 1. An overview of the reduced representation hydroxymethylation profiling (RRHP) pipeline that has been used

\section{ACKNOWLEDGMENT}

This study has received funding from the European Research Council (ERC) under the European Union's Horizon 2020 research and innovation programme [grant agreement no 683210] and from the Research Council of Norway under the Toppforsk programme [grant agreement no 250548/F20]. Fedor Sharko was supported by the RFBR (Russian Foundation for Basic Research) Grant no 19-04-00033. 